\title{
Retained Foreign Body After Stingray Injury; A Case Report
}

(1DŞeref Emre Atiş' ${ }^{1}$ (1) Bora Çekmen'1, (1) Öner Bozan²

'Karabuk University, Faculty of Medicine, Department of Emergency Medicine, Karabuk, Turkey

${ }^{2}$ Prof. Dr. Cemil Tascioglu City Hospital, Department of Emergency Medicine, Istanbul, Turkey

\begin{abstract}
Stingray is a demersal sea fish that causes widespread injury around the world. Stingray envenomation may cause local tissue injury as well as systemic effects. Although immersion of the injured area in hot water is effective in the treatment, this treatment does not provide complete relief in cases where there is a foreign body in the tissue. We presented about a case of a 22 -year-old male patient with stingray tail detected in $\mathrm{x}$-ray images taken after complete relief was not provided after treatment.

Keywords: stingray,injuries,foreign objects,envenomation
\end{abstract}

\section{Introduction}

Sea stingray is a deep fish species that is common in the world and has a variable number of stings on its tail and venomous glands ${ }^{1}$. The injury is caused by the mechanical effect of the tail, as well as the serotonin, phosphodiesterase containing poison in the gland. Serotonin content is responsible for pain in individuals, and phosphodiesterase derivatives are responsible for local tissue destruction ${ }^{2}$. Injury may remain local or systematic symptoms could be seen such as nausea, vomiting, diarrhea, rabdomyolysis, cardiac injury ${ }^{3}$. Injury is mostly related to foot or lower extremity ${ }^{4}$. We present a case report who was brought to our emergency department after a marine stingray injury and afterwards a retained stingray fragment was found.

\section{Case Report}

A 22-year-old male patient, who has no known disease, was admitted to the emergency room after stingray envenomation from his foot while cleaning the fishing net. He had no history of allergy and his vital signs were stable when he arrived. The patient had regional edema and an approximately $1 \mathrm{~cm}$ laceration in the dorsolateral region of the right foot and a bullous injury approximately $3 \mathrm{~cm}$ size above the laceration (Picture 1). There was no motor or sensory loss in the right foot. Initially hot water immersion treatment performed but when the pain was remaining, opioid-derived painkillers added for analgesia. He complained of severe pain again after a while during the follow-up. A radiopaque foreign body (stingray tail) was found near the 5th metacarpal bone in the $\mathrm{x}$-ray radiographs of the patient (Picture 2). In laboratory creatine kinase was $377 \mathrm{U} / \mathrm{L}$ (normal range 60-300 U/L), aspartate aminotransferase was $139 \mathrm{U} / \mathrm{L}$, alanine aminotransferase was $160 \mathrm{U} / \mathrm{L}$ (normal values 15-42, 15-50 U/L, respectively) and other values were in the normal range. The patient was consulted with Orthopedics. The foreign body in the foot was removed with local anesthesia, and the surrounding tissue was debrided. The patient was hospitalized for follow-up and antibiotic treatment, was discharged 3 days later with full recovery.

\section{Discussion}

Although stingray envenomation can occur anywhere on the body depending on the area of contact, it is mostly seen in lower extremities. In a prospective study of 22 patients, the injury site was mostly determined as the plantar foot region, but in our case the dorsolateral area of the foot was injured. In the treatment of stingray envenomation, immersion the injured area in hot water near 30-90 minutes neutralizes the effects of the labil poison and provides relief of symptoms ${ }^{2}$. In addition to immersion in hot water, we added an opioid analgesic, as the patient's pain was not fully resolved. In a retrospective study of Clark et al. there was no stingray part was found in the foot on the imaging performed after injury.

Corresponding Author: Şeref Emre Atiş e-mail: dremreatis@gmail.com

Received: 18.10.2021 • Accepted: 10.12.2021

Cite this article as: Atis SE, Cekmen B, Bozan O. Retained foreign body after stingray injury; a case report. Eurasian J Tox. 2021;3(3):56-57. 


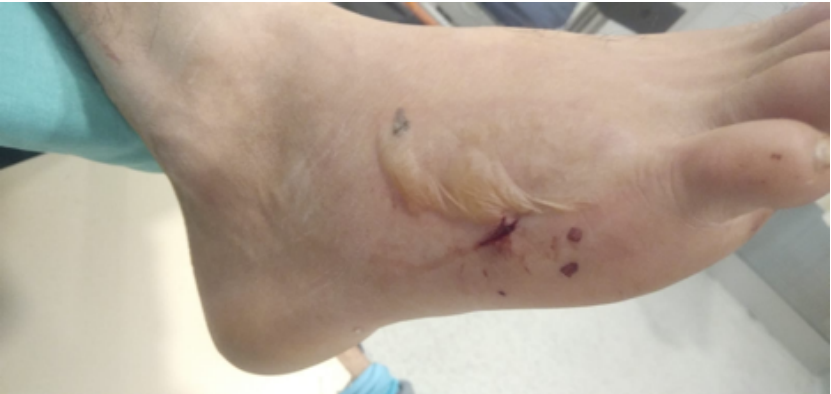

Picture 1. The patient had regional edema and an approximately $1 \mathrm{~cm}$ laceration in the dorsolateral region of the right foot and a bullous injury approximately $3 \mathrm{~cm}$ size above the laceration

Suspicious imaging was provided in only one case, and no structure other than sand was detected in the wound exploration. In the same study, symptomatic reprieve was observed in all patients by immersing the affected limb in hot water and some patients with additional painkillers ${ }^{3}$. In another prospective study of 22 patients, no foreign body was detected after the envenomation and all patients' pain were relieved by hot water treatment or hot water plus povidin iodine application $^{5}$. In the literature, there are only few cases which reported positive radiological imaging. ${ }^{6,7}$. In our case, the tail of the stingray was detected on $\mathrm{x}$-ray imaging, which is rare in the literature, and this foreign body was removed and wound was debrided. Evans et al. was stated that the foreign body embedded in the wound site caused the absence of symptomatic relief and tissue necrosis ${ }^{8}$. In our case, pain relief was not achieved after the treatment and a tail part of the stingray was found close to the entrance with imaging techniques.

In envenomation of stingray, which is a common marine vertebrae in the coastal areas, patients who cannot be relieved by conventional treatment, retained foreign body (especially the part of the stingray tail) should be suspected and local tissue exploration with diagnostic imaging techniques are required.

\section{References}

1. Otten EJ, Blomkalns AL. Venomous animal injuries. In:Marx JA, Hockberger RS, Walls RM et al., Emergen-
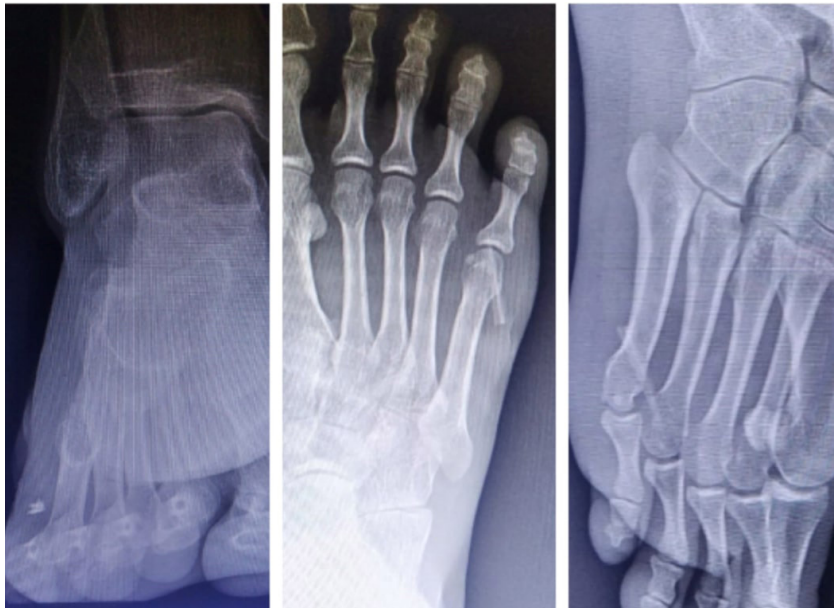

Picture 2: A radiopaque foreign body (stingray tail) was found near the 5th metacarpal bone in the $x$-ray radiographs of the patient.

cy Medicine: concept and clinical practice, 5th edition St.louis MO: Mosby; 2002:785-800.

2. Kline A. Stingray envenomation of the foot: a case report. Foot Ankle Online J 2008; 1:4.

3. Clark RF, Girard RH, Rao D, Ly BT, Davis DP. Stingray envenomation: a retrospective review of clinical presentation and treatment in 119 cases. The Journal of emergency medicine, 2007;33(1): 33-37.

4. Kamajian G, Singletary B Case Series: Stingray Envenomation. J. Urgent Care Med. 2014

5. Myatt $\mathrm{T}$, Nguyen BJ, Clark RF, Coffey $\mathrm{CH}$, O'Connell, CW A prospective study of stingray injury and envenomation outcomes. The Journal of emergency medicine 2018;55(2):213-217.

6. Cook MD, Matteucci MJ, Lall R, Ly BT. Stingray envenomation. Journal of Emergency Medicine 2006;30(3): 345-347.

7. Moyles BG, Wilson RC. Stingray spine foreign body in the foot. The Journal of foot surgery. 1989;28(1):30-32.

8. Evans RJ, DaviesRS. Stingray Injuries Emergency Medicine Journal 1996;13(3):224-225. 